\title{
Relationship of Psychosocial Safety Climate and Workplace Psychosocial Risks: A Randomized Trail among Personnel of an Oil and Gas Company in Iran
}

\author{
Dordaneh Amiri ${ }^{1}$, Lye Munn Sann ${ }^{1}$, Mohd Yusoff Adon ${ }^{2}$, Hjh Firdaus Mukhtar ${ }^{1}$, Khairuddin Idris ${ }^{3}$, Karl Kuhn ${ }^{4}$ \\ \& Ali Abbas Kamari ${ }^{5}$ \\ ${ }^{1}$ Faculty of Medicine and Health Science, University Putra Malaysia, Malaysia \\ ${ }^{2}$ Institute of Medical Research, Ministry for Health Malaysia, Malaysia \\ ${ }^{3}$ Faculty of Education, University Putra Malaysia, Malaysia \\ ${ }^{4}$ Federal Institute for Occupational Safety and Health, Germany \\ ${ }^{5}$ School of Humanities, Tarbiat Modares University, Iran \\ Correspondence: Lye Munn Sann, Faculty of Medicine and Health Science, University Putra Malaysia, Malaysia. \\ Tel: 603-8947-2410. E-mail: lyems9@yahoo.com
}

Received: October 11, 2014 Accepted: December 30, 2014 Online Published: April 30, 2015

doi:10.5539/ass.v11n12p69 URL: http://dx.doi.org/10.5539/ass.v11n12p69

\begin{abstract}
Oil and gas industry is a main revenue generation for Iran, and thousands of employees are involved in various oil and gas fields. From numerous health hazards which commonly occur in the industry one of them is psychosocial hazards. Studies confirmed a crucial step to control and understanding workplace psychosocial risk factors is developing and monitoring of policies, rules, and plans to promote health at work. This research conducted in an Iranian oil and gas company in Tehran in the period of 2011 and 2012. 248 participants were randomly selected and assigned in the study. Two questionnaires HSE and SCAT used in order to assess workplace psychosocial risks and attitude of personnel to safety climate. The result of HSE scores revealed relationships and role sub-scales in psychosocial risks were in abysmal situation. Personnel's attitude to safety climate at the organization displayed communication, priority of safety, and involvement were at the level of "dissatisfied". Also there was a medium positive correlation between work environment and managers' support, relationships and change $\mathrm{p}<0.01$. These findings show that safety climate factors namely communication, work environment, supportive environment, inversely affects workplace psychosocial risks. Overall, the outcomes supported the possibility that personnel attitudes to safety climate at company were predictive of higher psychosocial risks. Progress in safety climate and the psychosocial aspects of safety climate may diminish the experience psychosocial risks. Development of policies and procedures with aim of reduce psychosocial risks should be considered in Iran.
\end{abstract}

Keywords: safety climate, psychosocial risk, oil and gas industry, HSE tool, SCAT tool

\section{Introduction}

Since 1920 oil and gas industry has become the main revenue generation for Iran (Mohamedi, 2011). National Iranian Oil Company (NIOC) reported thousands of employees are involved in various oil and gas fields (NIOC, 2012).

One of the high-risk occupations in terms of health, safety, and environment is working in the field of oil and gas industry. Numerous hazards which commonly occur in the industry involve chemicals (toxic, sensitising substances), physical concerns (noise, vibration), biological effects (food poisoning), ergonomic activities (manual handling methods), and psychosocial impacts (work overload, long working hours, work relationships) (Gardner, 2003). Psychosocial risks factors are "elements that impact employees' psychological responses to work and work conditions, potentially causing psychological health problems" (Samra, Gilbert, Shain, \& Bilsker, 2009).

There are three different workplace situations in the oil and gas industry, including oil fields, laboratories, and offices, which expose employees to different health risks. Research findings show addressing and dealing with 
psychosocial risks is a challenge for managers and occupational health and safety inspectors (Productivity Commission, 2010) (Johnstone, Quinlan, \& McNamara, 2010). Studies found an essential step to control and understanding workplace psychosocial risk factors is developing and monitoring of policies, rules, and plans to promote health at work (Dollard, Skinner, Tuckey, \& Bailey, 2007).

Research related to workplace psychosocial conditions in developing countries are not solid and organized (Kortum, Leka, \& Cox, 2010). Identifying the magnitude of correlation between psychosocial risk factors, would provide stakeholders, government, management, health and safety professionals, and health personnel, an opportunity to address psychosocial risks appropriately and effectively.

Attitudes and perceptions of employees to the workplace makes safety climate (Carayon, 2007), and Psychosocial safety climate is policies, rules and procedures to protect worker from psychological health and safety problems in the organization (Dollard \& Bakker, 2010).

There is significant evidence that psychosocial risks can lead to physical and mental disease (Cheng, Kawachi, Coakley, Schwartz, \& Colditz, 2000; Cooper \& Cartwright, 1994; Cooper \& Sutherland, 1987; Kazi \& Haslam, 2013; LaRocco, House, \& French Jr., 1980; Leka \& Jain, 2010; Portuné, 2012; Schmidt, Roesler, Kusserow, \& Rau, 2014; Wang \& Schmitz, 2011). (Choobineh, Movahed, Tabatabaie, \& Kumashiro, 2010; HSE, 2008; Lee, Yeh, Chen, \& Wang, 2005; Portuné, 2012).

Some models like the Palmer and Cooper model (2001), attempts to show a scientific association between psychosocial risk factors, perceived as demands, control, role, change, relationships, and support, with symptoms of stress in employees, to which the organisational culture also contributes to stress levels, all of which have negative outcomes.

Dollard and Bakker considered the PSC was an important organisational resource, which influenced demands and resources. The psychosocial safety climate model demonstrated that a lack of policies and procedures to contain work demands, could lead to work pressures and fatigue, and increase the necessity to hide emotions at the workplace. If employers do not pay attention to employee's concerns, or ignore psychological well-being at work, the effects would be detrimental (Dollard \& Bakker, 2010).

Dollard predicted the psychosocial safety climate was an important factor in workplace mental health, and job resources, such as social support, performance feedback, and autonomy, may instigate a motivational process leading to job-related learning, work engagement, and organisational commitment (Dollard \& Bakker, 2010).

Many scientific research findings suggest that psychosocial factors play an important role in the development of mental health problems (Chang et al., 2006; Chen, Wong, \& Yu, 2009; Nomura, Nakao, Sato, Ishikawa, \& Yano, 2007). Therefore psychosocial risks were recognised as an emerging occupational health and safety priority risk, by the European Commission's Strategy on Health and Safety at Work 2002 - 2006 (Dollard et al., 2007).

The result of a cross-sectional survey to measure the relationship of job stress and mental health in Chinese offshore oil platform workers, demonstrated a significant association between mental health problems and occupational stress. Generally, findings emphasise reducing occupational stress, to promote workplace mental health (Chen et al., 2009). Cooper and Sutherland studies have demonstrated that employees working offshore have more anxiety than the general population, and a predictor of anxiety in offshore workers was stress from work and home relationships (Chen et al., 2009; Cooper \& Sutherland, 1987).

Research conducted in a thermal power plant in China about the effects on workers' well-being of job demands, control, and reward, found that employees who reported high levels of work demands and low levels of decision making, or high efforts and low rewards, raised the risk of job dissatisfaction, psychosomatic complaints, and depressive symptoms (Yu, Gu, Zhou, \& Wang, 2008).

A study on occupational mental health among Caribbean nurses showed that role conflict, role overload, and social support, were related to stress and burnout. Burnout was the sole predictor of depression, which in turn was responsible for both absenteeism and loss of turnover (Baba, Galperin, \& Lituchy, 1999). The 2003 study by Michie \& Williams cited in (Dollard \& McTernan, 2011), reviewed scientific papers written from 1987 to 1999 on work-related psychological ill-health in the health care sector and clarified that demands and low levels of control, decision making, social support, role conflicts, and unclear management, were the most significant stressors.

Studies in Europe found that the creation of new rule structures by occupational health services at the workplace, will reduce the difficulty of dealing with all aspects of occupational health risks (Cox, Leka, Ivanov, \& Kortum, 2004). 
Consequently, there is a necessity to enhance the skills of occupational health and safety personnel, so they know how to counter workplace psychosocial risk (Ylikoski, 2008). The involvement of employees plays a key role in preparing meaningful health programmes. The main elements of comprehensive health promotion at the workplace, lay a strong emphasis on psychosocial factors that affect workers' health, and the participation of employees in the process of identifying problems and developing suggestions for improvement (Aust \& Ducki, 2004). The promotion and prevention programmes, will attempt to create a climate that fosters motivation and commitment, reduces obvious stressful agents, and promotes harmony among co-workers. Studies illustrate the importance of health education, in order to increase awareness of factors affecting mental health, well-being, and detecting risk factors (Harnois \& Gabriel, 2000).

\section{Method}

Data and measure:

Research conducted in an oil and gas company which working in the field of Engineering Procumbent Construction (EPC) with 1,200 employees.

The sample was taken from all departments of the company in the period of 2011 and 2012. The Human Resources and Communications (HR \& C) department delivered the list of employees in Microsoft Excel format that included updated demographic data.

The First inclusion criteria were Iranian. Then by age factor, employees under 25 years old age was filtered. Based on sample size formula 248 out of 714 were eligible. Questionnaires were a macro in Microsoft excel, which guide participants to use and fill the questionnaires.

An instrument was HSE management standards indicator tool developed by The Health and Safety Executive (HSE) of U.K. to evaluate psychosocial risks at the workplace. It contains 35 questions which specify how the respondent is performing in seven risks based on a five-point Likert scale. 23 questions responses are from never to always and 12 questions from strongly disagree to strongly agree. A lower score shows poor performance, or a potential problem area. The scores range from 1 to 5 (HSE, 2004). This questionnaire measures seven HSE risks at the workplace: Demands, Control, Managerial Support, Peer Support, Relationships, Role and Change.

Safety climate assessment tool (SCAT) was developed by Centre for Hazard and Risk Management at Loughborough University It measures staff responses across nine dimensions which are: Management commitment to safety, Communication, Priority of safety, Safety rules and procedures, Supportive environment, Involvement, Work environment, Personal priorities and need for safety, Personal appreciation of risk (Cox, \& Cheyne 2000).

The questionnaire has 43 items. Questions 1-7 reflect the subscale of management commitment scale. Communication is indicated by questions $8-12$, priority of safety (questions 13-16), safety rules and procedures (questions 17-19), supportive environment (questions 20-25), involvement (questions 26-28), personal priorities and need for safety (questions 29-33), personal appreciation of risk (questions 34-37), and work environment (questions 38-43). Each item was scored by giving a value of 5 to the 'strongly agree' category, 4 to the 'agree' response, 3 to the 'neither agree nor disagree' category, 2 to the 'disagree' response, and 1 to the 'strongly disagree' category. Six is the cut-off point to determine satisfaction of the employee (Loughborough University 1998)

Upon completion of the field survey and data collection (answers of two questionnaires), data were transferred from Microsoft Excel into IBM SPSS for statistical analysis. Statistical analyses were performed using IBM SPSS version 21 and Microsoft Excel 2010.

\section{Result}

According to inclusion and exclusion criteria, from 1193 staff, 714 were eligible and 248 personnel volunteered for research. 206 (83.1\%) personnel completed questioners.

\subsection{HSE Management Indicator}

Seven risks measured by The HSE management indicator (Demands, Control, Managers' support, Peer support, Relationship, Role, and Change).

Result revealed Relationship and role displayed "Urgent action needed" with means of 3.48, and 4.36 respectively, while the mean scores for demands, control, managers' support, peer support and change indicated "clear need for improvement", with values of 3.10, 3.10, 3.17, 3.57, and 3.07 respectively. 
Table 1. Socio-demographic characteristics

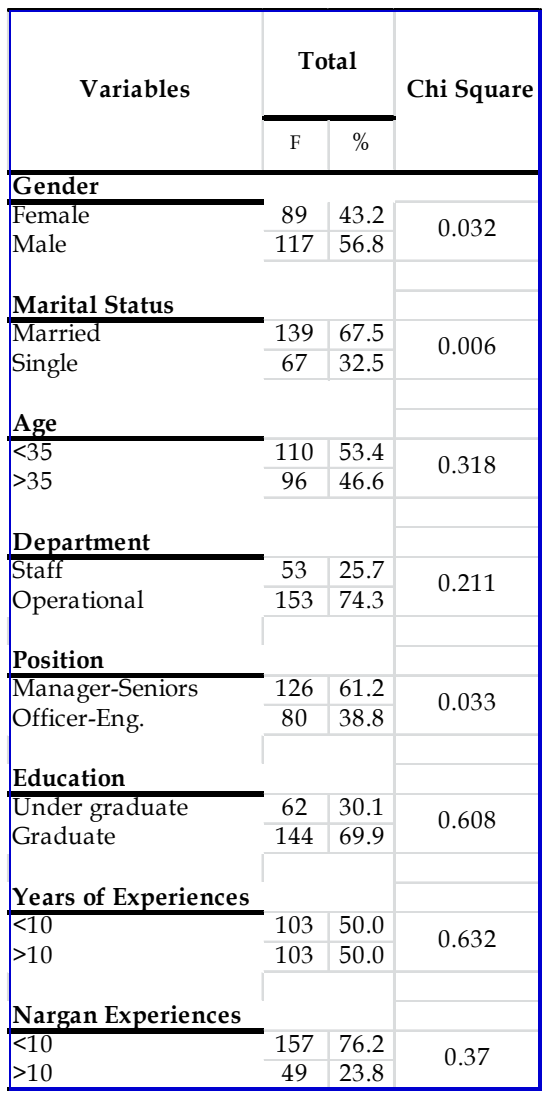

Table 2. Means, Standard Deviation for HSE factors scores and distribution of employees by levels of action needed

\begin{tabular}{|c|c|c|c|c|c|c|c|c|c|c|}
\hline \multirow{2}{*}{$\begin{array}{l}\text { HSE risk } \\
\text { Sub-scales }\end{array}$} & \multirow[t]{2}{*}{ Mean } & \multirow[t]{2}{*}{ SD } & \multicolumn{2}{|c|}{$\begin{array}{l}\text { Urgent action } \\
\text { needed }\end{array}$} & \multicolumn{2}{|c|}{$\begin{array}{l}\text { Clear need for } \\
\text { improvement }\end{array}$} & \multicolumn{2}{|c|}{$\begin{array}{l}\text { Good, but need for } \\
\text { improvement }\end{array}$} & \multicolumn{2}{|c|}{$\begin{array}{l}\text { Doing very well - need } \\
\text { to maintain performance }\end{array}$} \\
\hline & & & $\mathrm{F}$ & $\%$ & $\mathrm{~F}$ & $\%$ & $\mathrm{~F}$ & $\%$ & $\mathrm{~F}$ & $\%$ \\
\hline Demands & 3.1 & 0.67 & 91 & 44.2 & 50 & 24.3 & 50 & 24.3 & 15 & 7.3 \\
\hline Control & 3.1 & 0.69 & 67 & 32.5 & 71 & 34.5 & 59 & 28.6 & 9 & 4.4 \\
\hline Managers' Support & 3.17 & 0.7 & 69 & 33.5 & 105 & 51 & 29 & 14.1 & 3 & 1.5 \\
\hline Peer Support & 3.57 & 0.67 & 79 & 38.3 & 53 & 25.7 & 63 & 30.6 & 11 & 5.3 \\
\hline Relationships & $3.48^{*}$ & 0.79 & 113 & 54.9 & 47 & 22.8 & 36 & 17.5 & 10 & 4.9 \\
\hline Role & $4.36^{*}$ & 0.53 & 111 & 53.9 & 60 & 29.1 & - & - & 35 & 17 \\
\hline Change & 3.07 & 0.71 & 42 & 20.4 & 103 & 50 & 27 & 13.1 & 34 & 16.5 \\
\hline
\end{tabular}

* Urgent action needed / - In the HSE manual there are no scores for section "Good, but need for improvement" in role scale.

The means and standard deviations for HSE factor scores discovered the dire conditions of two psychosocial risks that are relationships and role sub-scales. As table 2 shows, $54.9 \%$ were in "urgent action needed" level for relationships score and 53.9\% were in "urgent action needed" level for role scale.

Table 3 shows result of the different levels of socio-demographic factors. Females had higher demands score than males $(p=0.046)$. Those more than 35 years of age had higher peer support and change scores than those $<35$ ( $p=0.045$ and $p=0.002$ respectively). Those in staff departments had higher relationships scores than those in operational departments $(\mathrm{p}=0.049)$. "Managers- seniors" had higher demands scores than those in "officers-engineers" position $(\mathrm{p}=0.009)$. Those with $>10$ years of experience had higher scores for peer support, relationships and change $(\mathrm{p}=0.0035, \mathrm{p}=0.022$ and $\mathrm{p}=0.031$ respectively). Undergraduates had higher scores for relationships and role than graduate employees ( $\mathrm{p}=0.012$ and $\mathrm{p}=0.001$ respectively). 
Table 3. Mean scores for employees for each sub-scale compared across different socio-demographic factors

\begin{tabular}{|c|c|c|c|c|c|c|c|}
\hline \multicolumn{3}{|c|}{ HSE-Socio-demographic } & $\mathrm{N}$ & Mean & Std. & Std. Error & $P$ value \\
\hline \multirow{2}{*}{ Gender } & Female & \multirow{2}{*}{ Demands } & 89 & 3.21 & 0.676 & 0.072 & \multirow{2}{*}{0.046} \\
\hline & Male & & 117 & 3.02 & 0.659 & 0.061 & \\
\hline \multirow{4}{*}{ Age } & $\leq 35$ years of old & \multirow{2}{*}{ Peer Support } & 110 & 3.48 & 0.687 & 0.066 & \multirow{2}{*}{0.045} \\
\hline & $>35$ years of old & & 96 & 3.67 & 0.645 & 0.066 & \\
\hline & $\leq 35$ years of old & \multirow{2}{*}{ Change } & 110 & 2.94 & 0.716 & 0.068 & \multirow{2}{*}{0.002} \\
\hline & $>35$ years of old & & 96 & 3.23 & 0.677 & 0.069 & \\
\hline \multirow{2}{*}{ Department } & Staff & \multirow{2}{*}{ Relationships } & 53 & 3.67 & 0.835 & 0.115 & \multirow{2}{*}{0.049} \\
\hline & Operational & & 153 & 3.42 & 0.771 & 0.062 & \\
\hline \multirow{4}{*}{ Position } & Managers-Seniors & \multirow{2}{*}{ Demands } & 126 & 3.2 & 0.68 & 0.061 & \multirow{2}{*}{0.009} \\
\hline & Officers-Engineers & & 80 & 2.95 & 0.633 & 0.071 & \\
\hline & Managers-Seniors & \multirow{2}{*}{ Change } & 126 & 2.99 & 0.714 & 0.064 & \multirow{2}{*}{0.036} \\
\hline & Officers-Engineers & & 80 & 3.2 & 0.695 & 0.078 & \\
\hline \multirow{6}{*}{ 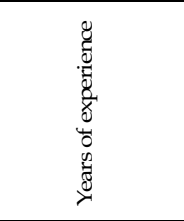 } & $\leq 10$ years of experience & \multirow{2}{*}{ Peer Support } & 103 & 3.47 & 0.689 & 0.068 & \multirow{2}{*}{0.035} \\
\hline & $>10$ years of experience & & 103 & 3.67 & 0.645 & 0.064 & \\
\hline & $\leq 10$ years of experience & \multirow{2}{*}{ Relationship } & 103 & 3.35 & 0.818 & 0.081 & \multirow{2}{*}{0.022} \\
\hline & $>10$ years of experience & & 103 & 3.61 & 0.75 & 0.074 & \\
\hline & $\leq 10$ years of experience & \multirow{2}{*}{ Change } & 103 & 2.97 & 0.676 & 0.067 & \multirow{2}{*}{0.031} \\
\hline & $>10$ years of experience & & 103 & 3.18 & 0.735 & 0.072 & \\
\hline \multirow{4}{*}{ Education level } & Under graduate & \multirow{2}{*}{ Relationships } & 62 & 3.69 & 0.752 & 0.095 & \multirow{2}{*}{0.012} \\
\hline & Graduate & & 144 & 3.39 & 0.796 & 0.066 & \\
\hline & Under graduate & \multirow{2}{*}{ Role } & 62 & 4.54 & 0.413 & 0.052 & \multirow{2}{*}{0.001} \\
\hline & Graduate & & 144 & 4.29 & 0.562 & 0.047 & \\
\hline
\end{tabular}

Generally, results shows many of the psychosocial risks specifically need to be improved.

\subsection{Safety Climate Assessment Tool (SCAT)}

Safety climate assessment tool (SCAT) measures satisfaction levels of employees in nine factors (management commitment, communication, priority of safety, safety rules and procedures, supportive environment, involvement, personal priorities and need for safety, personal appreciation of risk, and work environment). Management commitment, safety rules and procedures, supportive environment, personal priorities and need for safety, personal appreciation of risk, and work environment were at the level of "Satisfied" with means of 3.10, $3.10,3.17,3.57$, and 3.07 respectively. Communication, priority of safety, and involvement were at the level of "Dissatisfied" with means of 5.86, 5.66 and 5.99 respectively.

Table 4. Means, Standard Deviations for SCAT scores

\begin{tabular}{ccccc}
\hline SCAT sub-scales & Min & Max & Mean & S.D. \\
\hline Management Commitment & 3 & 10 & 6.48 & 1.07 \\
Communication & 2 & 8 & $5.86^{*}$ & 0.75 \\
Priority of Safety & 4 & 10 & $5.66^{*}$ & 0.81 \\
Safety Rules and Procedures & 4 & 10 & 6.34 & 0.99 \\
Supportive Environment & 5 & 8 & 6.75 & 0.68 \\
Involvement & 3 & 9 & $5.99^{*}$ & 0.89 \\
Personal Priorities and Need for Safety & 4 & 10 & 7.33 & 1.16 \\
Personal Appreciation of Risk & 4 & 10 & 6.66 & 1.05 \\
Work Environment & 4 & 10 & 6.03 & 1.2 \\
\hline
\end{tabular}

\section{*. Dissatisfied level}

Radar chart of means for SCAT (Figure 1), shows the "dissatisfied "levels of SCAT sub-scales compared to "satisfied" levels. From the index line $(\geq 6)$, we can see clearly which components are under index line and which ones are above.

Table 5. Distribution of employees' satisfaction level for SCAT scores

\begin{tabular}{|c|c|c|c|c|c|c|}
\hline \multirow{2}{*}{ SCAT Factors } & \multicolumn{2}{|c|}{ Communication } & \multicolumn{2}{c|}{ Priority of safety } & \multicolumn{2}{c|}{ Involvement } \\
\cline { 2 - 7 } & Frequency & Percent & Frequency & Percent & Frequency & Percent \\
\hline Dissatisfied & 86 & 41.7 & 117 & 56.8 & 74 & 35.9 \\
\hline Satisfied & 120 & 58.3 & 89 & 43.2 & 132 & 64.1 \\
\hline Total & 206 & 100 & 206 & 100 & 206 & 100 \\
\hline
\end{tabular}




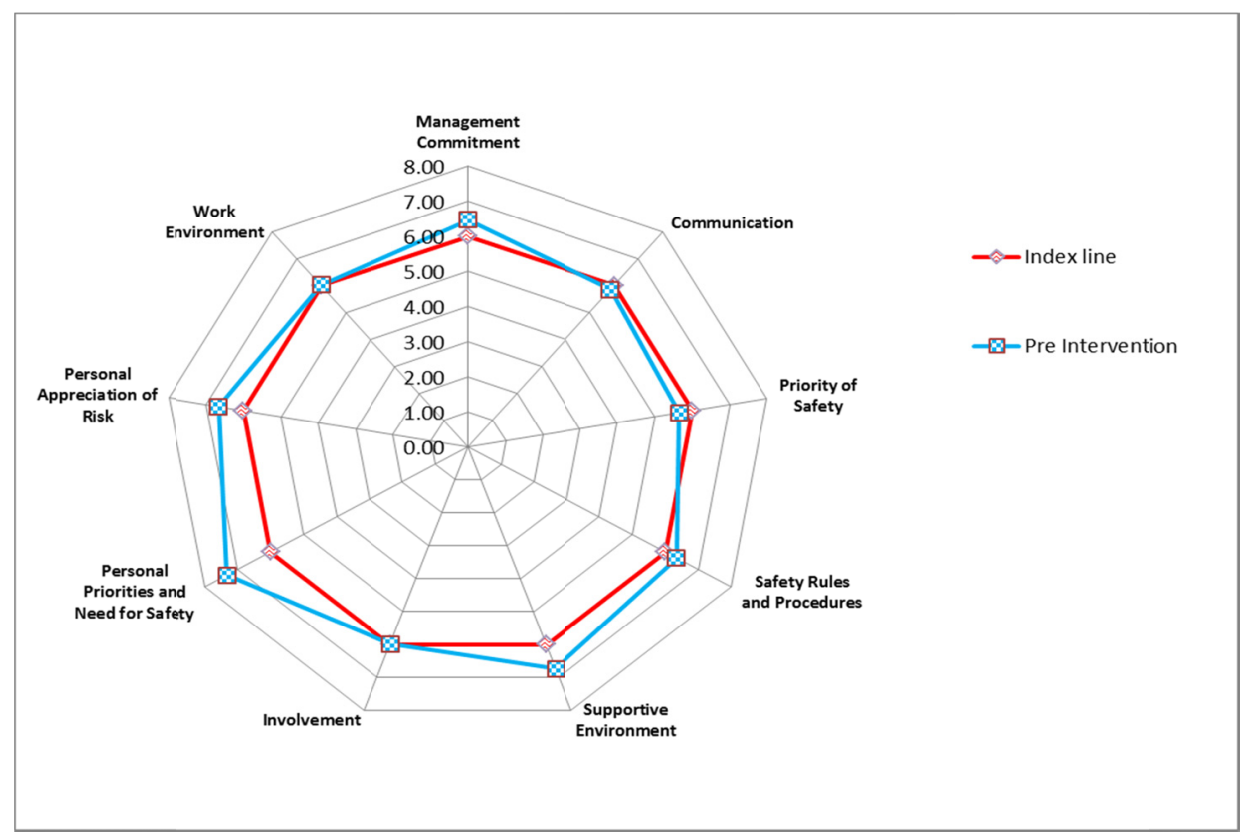

Figure 1. Radar chart of Means for SCAT

As table 5 shows for attitude of participants about safety climate in company showed that a high proportion of employees were dissatisfied with three safety climate factors, which are communication, priority of safety and involvement. Almost $42 \%$ participants were dissatisfied with communication in organization, and for Priority of safety factor almost $57 \%$ participants were dissatisfied, and $36 \%$ were dissatisfied with involvement in company.

Table 6 shows mean SCAT scores compared across different socio-demographic factors.

Table 6. SCAT - Socio-demographic

\begin{tabular}{|c|c|c|c|c|c|c|c|}
\hline & \multicolumn{2}{|c|}{ SCAT - Socio-demographic } & \multirow{3}{*}{\begin{tabular}{|c|}
$\mathrm{N}$ \\
110 \\
96
\end{tabular}} & \multirow{3}{*}{\begin{tabular}{|l|} 
Mean \\
6.32 \\
6.65 \\
\end{tabular}} & \multirow{3}{*}{\begin{tabular}{|c|c|}
$\begin{array}{c}\text { Std. } \\
\text { Deviation }\end{array}$ \\
1.003 \\
1.128 \\
\end{tabular}} & \multirow{3}{*}{\begin{tabular}{|c|}
$\begin{array}{c}\text { Std. Error } \\
\text { Mean }\end{array}$ \\
0.096 \\
0.115 \\
\end{tabular}} & \multirow{3}{*}{$\begin{array}{c}\text { P value } \\
0.027\end{array}$} \\
\hline \multirow{6}{*}{ Age } & $\leq 35$ years of old & \multirow{2}{*}{$\begin{array}{l}\text { Management } \\
\text { Commitment }\end{array}$} & & & & & \\
\hline & $>35$ years of old & & & & & & \\
\hline & $\leq 35$ years of old & \multirow{2}{*}{$\begin{array}{l}\text { Personal Priorities and } \\
\text { Need for Safety }\end{array}$} & 110 & 7.13 & 1.104 & 0.105 & \multirow{2}{*}{0.008} \\
\hline & $>35$ years of old & & 96 & 7.56 & 1.19 & 0.121 & \\
\hline & $\leq 35$ years of old & \multirow{2}{*}{ Work Environment } & 110 & 5.83 & 1.137 & 0.108 & \multirow{2}{*}{0.012} \\
\hline & $>35$ years of old & & 96 & 6.25 & 1.235 & 0.126 & \\
\hline \multirow{4}{*}{ 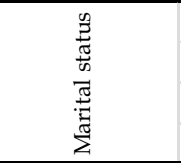 } & Married & \multirow{2}{*}{$\begin{array}{l}\text { Management } \\
\text { Commitment }\end{array}$} & 139 & 6.6 & 1.123 & 0.095 & \multirow{2}{*}{0.018} \\
\hline & Single & & 67 & 6.22 & 0.917 & 0.112 & \\
\hline & Married & \multirow{2}{*}{ Supportive Environment } & 139 & 6.82 & 0.625 & 0.053 & \multirow{2}{*}{0.028} \\
\hline & Single & & 67 & 6.6 & 0.756 & 0.092 & \\
\hline \multirow{8}{*}{ 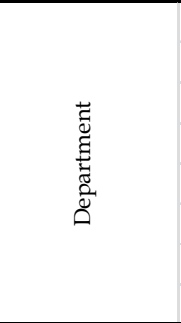 } & Staff & \multirow{2}{*}{$\begin{array}{l}\text { Management } \\
\text { Commitment }\end{array}$} & 53 & 6.81 & 1.058 & 0.145 & \multirow{2}{*}{0.007} \\
\hline & Operational & & 153 & 6.36 & 1.057 & 0.085 & \\
\hline & Staff & \multirow{2}{*}{$\begin{array}{l}\text { Safety Rules and } \\
\text { Procedures }\end{array}$} & 53 & 6.7 & 1.033 & 0.142 & \multirow{2}{*}{0.002} \\
\hline & Operational & & 153 & 6.21 & 0.944 & 0.076 & \\
\hline & Staff & \multirow{2}{*}{$\begin{array}{c}\text { Personal Appreciation of } \\
\text { Risk }\end{array}$} & 53 & 6.38 & 1.1 & 0.151 & \multirow{2}{*}{0.022} \\
\hline & Operational & & 153 & 6.76 & 1.02 & 0.082 & \\
\hline & Staff & \multirow{2}{*}{ Work Environment } & 53 & 6.47 & 1.227 & 0.169 & \multirow{2}{*}{0.002} \\
\hline & Operational & & 153 & 5.87 & 1.155 & 0.093 & \\
\hline \multirow{6}{*}{ 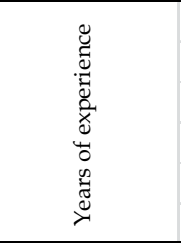 } & $\leq 10$ years of experience & \multirow{2}{*}{$\begin{array}{l}\text { Management } \\
\text { Commitment }\end{array}$} & 103 & 6.22 & 0.995 & 0.098 & \multirow{2}{*}{0.001} \\
\hline & $>10$ years of experience & & 103 & 6.73 & 1.093 & 0.108 & \\
\hline & $\leq 10$ years of experience & \multirow{2}{*}{$\begin{array}{l}\text { Personal Priorities and } \\
\text { Need for Safety }\end{array}$} & 103 & 7.16 & 1.133 & 0.112 & \multirow{2}{*}{0.035} \\
\hline & $>10$ years of experience & & 103 & 7.5 & 1.172 & 0.115 & \\
\hline & $\leq 10$ years of experience & \multirow{2}{*}{ Work Environment } & 103 & 5.78 & 1.118 & 0.11 & 0 \\
\hline & $>10$ years of experience & & 103 & 6.27 & 1.232 & 0.121 & 0.000 \\
\hline & Under graduate & Personal Priorities and & 62 & 7.6 & 0.885 & 0.112 & 0.024 \\
\hline & Graduate & Need for Safety & 144 & 7.2 & 1.246 & 0.103 & \\
\hline
\end{tabular}




\subsection{HSE Factors and SCAT Factors}

Table 7. HSE sub-scales and SCAT sub-scales

\begin{tabular}{|c|c|c|c|c|c|c|c|c|c|c|c|c|c|c|c|c|}
\hline HSE-SCAT & Demands & Control & $\begin{array}{l}\text { Managers } \\
\text { Support }\end{array}$ & Peer Support & Relationships & Role & Change & $\begin{array}{l}\text { Management } \\
\text { Commitment }\end{array}$ & Communication & $\begin{array}{c}\text { Priority of } \\
\text { Safety }\end{array}$ & $\begin{array}{c}\text { Safety Rules } \\
\text { and Procedures }\end{array}$ & $\begin{array}{l}\text { Supportive } \\
\text { Environment }\end{array}$ & Involvement & $\begin{array}{c}\text { Personal } \\
\text { Priorities and } \\
\text { Need for Safety }\end{array}$ & $\begin{array}{c}\text { Personal } \\
\text { appreciation of } \\
\text { Risk }\end{array}$ & $\begin{array}{c}\text { Work } \\
\text { Environment }\end{array}$ \\
\hline Demands & 1 & $.210^{* *}$ & 0.086 & 0.099 & $.185^{\prime \prime}$ & 0.006 & 0.08 & $.266^{\prime \prime}$ & 0.059 & -0.038 & 0.083 & $-.216^{\prime \prime}$ & 0.017 & $.204^{*}$ & 0.105 & $.447^{\prime \prime}$ \\
\hline Control & & 1 & $.361^{\circ}$ & $.315^{*}$ & 0.053 & $.174^{*}$ & $.379^{\prime \prime}$ & $.199^{*}$ & 0.106 & -0.004 & -0.001 & 0.015 & $.219^{\prime \prime}$ & 0.033 & 0.05 & $.195^{*}$ \\
\hline $\begin{array}{c}\text { Managers' } \\
\text { Support }\end{array}$ & & & 1 & $.487^{\prime \prime}$ & $.290^{\circ}$ & $.202^{*}$ & $.570^{*}$ & $.367^{*}$ & $.207^{\prime \prime}$ & 0.071 & -0.094 & 0.117 & $.185^{\prime \prime}$ & 0.062 & 0.122 & $.359^{\circ \prime}$ \\
\hline Peer Support & & & & 1 & $.381^{\prime \prime}$ & $.168^{*}$ & $.399^{\prime \prime}$ & $.226^{\prime \prime}$ & $.146^{*}$ & 0.088 & -0.101 & $.144^{*}$ & 0.056 & 0.111 & -0.002 & $.204^{\prime \prime}$ \\
\hline Relationship & & & & & 1 & $.195^{\prime \prime}$ & $.332^{\prime \prime}$ & $.317^{*}$ & $.297^{\prime \prime}$ & 0.107 & 0.052 & -0.009 & 0.032 & 0.072 & 0.013 & $.329^{\prime \prime}$ \\
\hline Role & & & & & & 1 & $.429^{\prime \prime}$ & $.157^{\circ}$ & 0.108 & 0.067 & 0.071 & -0.049 & $.151^{\circ}$ & $.254^{\prime \prime}$ & 0.058 & $.22^{*}$ \\
\hline Change & & & & & & & 1 & $349^{\prime \prime}$ & 0.092 & 0.118 & 0.041 & 0.095 & $.313^{\prime \prime}$ & $.172^{*}$ & $.189^{* \prime}$ & $.343^{\prime \prime}$ \\
\hline $\begin{array}{l}\text { Management } \\
\text { Commitment }\end{array}$ & & & & & & & & 1 & $.246^{\prime \prime}$ & $.221^{*}$ & 0.042 & -0.093 & $.301^{1}$ & $.260^{*}$ & 0.127 & $.544^{\prime \prime}$ \\
\hline $\begin{array}{c}\text { Communicati } \\
\text { on }\end{array}$ & & & & & & & & & 1 & 0.079 & -0.017 & $-283^{*}$ & 0.084 & -0.059 & -0.054 & $.174^{*}$ \\
\hline $\begin{array}{c}\text { Priority of } \\
\text { Safety }\end{array}$ & & & & & & & & & & 1 & -0.024 & -0.041 & -0.034 & -0.071 & 0.042 & 0.125 \\
\hline $\begin{array}{l}\text { Safety Rules } \\
\text { and } \\
\text { Procedures }\end{array}$ & & & & & & & & & & & 1 & 0.064 & -0.028 & $.332^{* *}$ & 0.046 & 0.132 \\
\hline \begin{tabular}{c|} 
Supportive \\
Environment
\end{tabular} & & & & & & & & & & & & 1 & -0.089 & 0.115 & 0.098 & -0.132 \\
\hline Involvement & & & & & & & & & & & & & 1 & $.173^{*}$ & 0.012 & $.186^{\prime \prime}$ \\
\hline $\begin{array}{c}\text { Personal } \\
\text { Prio rities and } \\
\text { Need for } \\
\text { Safety }\end{array}$ & & & & & & & & & & & & & & 1 & $.184^{*}$ & $.318^{*}$ \\
\hline $\begin{array}{c}\text { Personal } \\
\text { Appreciation } \\
\text { of Risk }\end{array}$ & & & & & & & & & & & & & & & 1 & $.311^{\prime \prime}$ \\
\hline $\begin{array}{c}\text { Work } \\
\text { Environment }\end{array}$ & & & & & & & & & & & & & & & & 1 \\
\hline
\end{tabular}

**. Correlation is significant at the 0.01 level (2-tailed)

*. Correlation is significant at the 0.05 level (2-tailed)

Table 7 shows that small positive correlations were found between Demands and SCAT sub-scales namely Management commitment, $r=0.266, p<0.01$, Personal Priorities and Need for Safety, $r=0.204, p<0.01$. Small negative correlations were seen between Demands and Supportive Environment, $r=-0.216, p<0.01$, and almost a large correlations were discovered between Demands and Work Environment, $r=0.447, p<0.01$,

Small positive correlations were found between Control and SCAT sub-scales: Management commitment, $r=0$. 199, $\mathrm{p}<0.01$, Involvement, $\mathrm{r}=0.219, \mathrm{p}<0.01$, Work Environment, $\mathrm{r}=0.195, \mathrm{p}<0.01$,

Small positive correlations were found between Managers' support and SCAT sub-scales: Communication, $r=0$. 207, $\mathrm{p}<0.01$, Involvement, $\mathrm{r}=0.185, \mathrm{p}<0.01$, Moderate positive correlations were found between Managers' support and: Management commitment, $r=0.367, p<0.01$, Work Environment, $r=0.359, p<0.01$,

Small positive correlations were found between Peer support and SCAT sub-scales: Management commitment, $r$ $=0.226, p<0.01$, Communication, $r=0.146, p<0.05$, Work Environment, $r=0.204, p<0.01$,

Moderate positive correlations were found between Relationship and: Communication, $r=0.297, p<0.01$, Management commitment, $r=0.317, p<0.01$, Work Environment, $r=0.329, p<0.01$,

Small positive correlations were found between Role and SCAT sub-scales: Management commitment, $r=0$. $157, p<0.05$, Involvement, $r=0.151, p<0.05$, Personal Priorities and Need for Safety, $r=0.254, p<0.01$, Work Environment, $\mathrm{r}=0.212, \mathrm{p}<0.01$,

Small positive correlations were found between Change and SCAT sub-scales: Personal Priorities and Need for Safety, $r=0.172, p<0.05$, Personal Appreciation of Risk, $r=0.189, p<0.01$, Moderate positive correlations were found between Change and: Management commitment, $r=0.349, p<0.01$ Involvement, $r=0.313$, $\mathrm{p}<0.01$ Work Environment, $\mathrm{r}=0.343, \mathrm{p}<0.01$,

\subsubsection{Effect of Safety Climate Factors on Psychosocial Risk Factors}

The multiple linear regression shows that "Supportive environment" and "work environment" scores significantly and inversely affects demands scores $(\mathrm{p}=0.003, \mathrm{p}=0.001)$ respectively. "Involvement" scores significantly and inversely affects control scores $(\mathrm{p}=0.014)$. "Management commitment", Supportive environment", "Communication", and "Work environment" scores significantly and inversely affects manager's support scores $(\mathrm{p}=0.013, \mathrm{p}=0.012, \mathrm{p}=0.001, \mathrm{p}=0.001$ respectively). 
Table 8. Multiple linear regression for HSE scores with SCAT sub-scales

\begin{tabular}{|c|c|c|c|c|c|c|}
\hline HSE factors & SCAT sub-scales & B & Std. Error & Beta & $\mathrm{t}$ & p-value \\
\hline \multirow{2}{*}{ Demand } & Supportive Environment & -.194 & .065 & -.196 & -2.977 & $0.003^{*}$ \\
\hline & Work Environment & .224 & .044 & .399 & 5.027 & $0.001^{*}$ \\
\hline Control & Involvement & .139 & .056 & .180 & 2.473 & $0.014^{*}$ \\
\hline \multirow{4}{*}{ Manager's support } & Management Commitment & .129 & .052 & .198 & 2.502 & $0.013^{*}$ \\
\hline & Communication & .155 & .062 & .167 & 2.515 & $0.012^{*}$ \\
\hline & Supportive Environment & .249 & .068 & .241 & 3.674 & $0.001^{*}$ \\
\hline & Work Environment & .160 & .046 & .275 & 3.462 & $0.001^{*}$ \\
\hline \multirow{4}{*}{ Peer support } & Communication & .134 & .064 & .150 & 2.106 & $0.036^{*}$ \\
\hline & Safety Rules and Procedures & -.112 & .048 & -.165 & -2.337 & $0.020^{*}$ \\
\hline & Supportive Environment & .228 & .070 & .229 & 3.243 & $0.001^{*}$ \\
\hline & Work Environment & .094 & .048 & .168 & 1.969 & $0.05^{*}$ \\
\hline \multirow{2}{*}{ Relationships } & Communication & .257 & .073 & .243 & 3.537 & $0.001^{*}$ \\
\hline & Work Environment & .169 & .054 & .255 & 3.098 & $0.002^{*}$ \\
\hline Role & Personal Priorities and Need for Safety & .105 & .036 & .229 & 2.918 & $0.004^{*}$ \\
\hline \multirow{3}{*}{ Change } & Supportive Environment & .172 & .070 & .163 & 2.450 & $0.015^{*}$ \\
\hline & Involvement & .197 & .053 & .245 & 3.691 & $0.001^{*}$ \\
\hline & Work Environment & .115 & .048 & .194 & 2.408 & $0.017^{*}$ \\
\hline
\end{tabular}

"Communication", "safety rules and procedures" supportive environment", and "work environment" scores significantly and inversely affects peer support scores $(p=0.036, p=0.020, p=0.001, p=0.05$ respectively). "Management commitment and "Work environment" scores significantly and inversely affects relationships scores $(\mathrm{p}=0.001, \mathrm{p}=0.002$ respectively). "Personal priority and need for safety" scores significantly and inversely affects role scores $(\mathrm{p}=0.004)$. "Supportive environment", "involvement", and "work environment" scores significantly and inversely affects change scores $(\mathrm{p}=0.015, \mathrm{p}=0.001, \mathrm{p}=0.017$ respectively). (Table 8$)$

\subsubsection{Effect of Psychosocial Risk Factors on Safety Climate}

Table 9. Multiple linear regression for safety

\begin{tabular}{|c|c|c|c|c|c|c|}
\hline SCAT Factors & HSE sub-scales & $\mathrm{B}$ & Std. Error & Beta & $\mathrm{t}$ & $\mathrm{p}$-value \\
\hline \multirow{3}{*}{ Management commitment } & Demands & .322 & .103 & .202 & 3.124 & .002 \\
& Manager's Support & .341 & .125 & .222 & 2.728 & .007 \\
\cline { 2 - 7 } & Relationships & .238 & .096 & .176 & 2.482 & .014 \\
\hline \multirow{2}{*}{ Communication } & Manager's Support & .197 & .094 & .183 & 2.094 & .038 \\
\hline & Relationships & .276 & .072 & .291 & 3.817 & .001 \\
\hline Supportive environment & Demands & -.231 & .071 & -.230 & -3.250 & .001 \\
\hline Involvement & Change & .367 & .114 & .294 & 3.210 & .002 \\
\hline \multirow{2}{*}{ Personal Priorities and Need for Safety } & Demands & .386 & .120 & .223 & 3.208 & .002 \\
\hline \multirow{2}{*}{ work environment } & Role & .507 & .162 & .233 & 3.135 & .002 \\
\hline & Demands & .712 & .106 & .399 & 6.706 & .000 \\
\hline & Relationships & .234 & .099 & .155 & 2.374 & .019 \\
\hline
\end{tabular}

\subsection{Climate Scores with HSE Sub-Scales}

The multiple linear regression shows that "demand", "manager's support" and "relationships" scores significantly and inversely affects management commitment scores $(p=0.002, p=0.007$, and 0.014 respectively). "Manager's support" and "relationships" scores significantly and inversely affects communication scores ( $p=0.037$, and 0.001 respectively). "Demands" scores significantly and inversely affects supportive environment scores $(p=0.001)$. "Change" scores significantly and inversely affects involvement scores $(p=0.002)$. "Demands" and "role" scores significantly and inversely affects personal priorities and need for safety scores $(p=0.002)$. "Demands" and "relationships" scores significantly and inversely affects work environment ( $\mathrm{p}=0.001$ and $\mathrm{p}=0.019$ respectively) (Table 9). 


\section{Discussion}

\subsection{Workplace Psychosocial Risk Factors using the HSE Management Standards Indicator Tool}

The HSE Management Standards Indicator Tool has two levels which describe acceptable conditions (Doing very well: need to maintain performance; and Good: but needs improvement). The other two levels signified a dangerous and unacceptable degree of risk in the workplace (Clear: need for improvement; and Urgent: action required).

The results showed that "relationships" and "role" factor needed urgent attention to recover. There are numerous reasons that are connected to disappointing relationships at work. One possible explanation is that because job security effect on relationships (Landsbergis, Grzywacz, \& LaMontage, 2012), while unemployment rate is high in Iran( around $11.5 \%$ in 2011) (Press.TV, 2012). Therefore the anxiety of having stability of employment for work life may cause workers allow unkind behaviours. Also a workplace environment without trust could set relationships in trouble (Cooper \& Cartwright, 1994).

Ineffective communication, impact low relationship at workplace (Fairhurst, 1993; Fairhurst \& Chandler, 1989; Sias, 2005). "Communication factor," in current research was at a level dissatisfaction and may lead to increase psychosocial risk.

Although most previous studies focused on demand and control as psychosocial risks at the workplace but reviewing studies of Schuster, Kessler \& Aseltine (1990), and Spector and Jex's study in 1998, as cited in (Inoue \& Kawakami, 2010), and (Fujiwara, Tsukishima, Tsutsumi, Kawakami, \& Kishi, 2003; Tsuno et al., 2009) found relationships is an strong factor on predicting stress in the workplace whereas there is a paucity of study about workplace relationships.

The "role" factor was another psychosocial risk in current research, it showed duties in the workplace are not quite clear and should be developed. The correlation between "relationships" and "role" $(\mathrm{r}=0.195, \mathrm{p}<0.01)$, pointed that clear roles will recover relationships. Deficiency of clarity in roles may affect an workplace's relationship by producing conflict among employees (Pomaki, Supeli, \& Verhoeven, 2007; Saijo, Ueno, \& Hashimoto, 2008).

In general, the result of this research was in-line with other researches, that underlined the necessity of improvement in workplace psychosocial risk factors specifically relationships (Fairhurst, 1993; Fairhurst \& Chandler, 1989; Sias, 2005).

\subsection{Prevalence of Workplace Safety Climate Using the Safety Climate Assessment Tool (SCAT)}

Excluding "communication, priority of safety and involvement" factors, it was found that participants were satisfied with and had a positive attitude to other safety climate factors (management commitment, safety rules and procedures, supportive environment, personal priorities, need for safety, personal appreciation of risk and work environment). A low score $(<6)$ for "communication" was reported in $41.7 \%$ of participants, which indicated communication between employees and managers was unsatisfactory; especially in regards to safety issues. It was discovered that managers were neither committed to bring safety information to the attention of personnel, nor to inform them of current concerns and issues within the workplace. In addition, employees did not receive praise for working safely.

Cohen et al. (1975), Smith et al. (1975) and Shannon et al. (1997) were in agreement that one important key factor that effected lower accident rates and safety performances was good communication between management and employees; whereby, supervisors gave information to workers regarding safety issues (Mearns, Whitaker, \& Flin, 2003). Therefore, when utilising the behavioural approach regarding safety within the workplace, workers were motivated to behave in a safe manner. An example would be when employees received praise for acting in a safe manner (Cox, Jones, \& Rycraft, 2004).

The results of this research reflected on how relationships and role scores, as workplace psychosocial risk factors, were connected and related to safety climate factors. A moderate, positive and significant correlation between the relationship score and the communication factor $(\mathrm{r}=0.297, \mathrm{p}<0.01)$ indicated that communication was related to interpersonal relationships. When employees' relationships were not exceptional, communication was unlikely to be at a satisfactory level.

A study proved that enhanced communication within work environments was linked to a decline in workplace violence; moreover, communication problems within the workplace resulted in unpleasant relationships (Hinkka, Kuoppala, Väänänen-Tomppo, \& Lamminpää, 2013). Enhanced communication in an organisation allowed the 
transmission of information and the interaction between employees to be managed easily (Peiró Silla, 2000). Therefore, communication affected relationships among subordinates and supervisors.

Dissatisfaction with the priority of the safety factor was expressed by $56.8 \%$ of participants. This implied that the management clearly considered the safety of employees as unimportant, and safety issues were not a high priority. Safety procedures were not carefully followed and the management did not consider safety to be of equal importance as the success of company projects.

A company with an excellent safety climate would allocate their highest priority to safety. Geller (1994) demonstrated few essential principles when practicing an enhanced safety climate, for example: involving employees in devising safety rules; using a behaviour-based approach within the company; employers focusing on achieving success and not on avoiding failure; employers paying attention to employees' self-esteem and empowering safety as a priority rather than a value (Choudhry, Fang, \& Mohamed, 2007). Employers should involve employees in appraising the calculation of safety within the organisation (Zohar \& Luria, 2003).

Managers should base their actions on safety rules, since the absence of such rules may lead employees to perceive the company as having a low safety priority. In addition, managers must recognise which safety issues should be of high priority and what behaviours are expected to be rewarded and reinforced. Zohar, as the founder of safety climates, explained that if output was more important than safety, employees will align their deeds in accordance to the detriment of safety (Zohar, 2010).

This study revealed that involvement was also at an unsatisfactory level, which played an important role in the workplace. Of the respondents, $35.9 \%$ were dissatisfied with the involvement factor in the company. It illustrated that those respondents were not involved in important safety issues at work and were not involved in the on-going review of safety.

Flin et al. (1996) confirmed the need to consider involvement of employees in safety-related decisions as a vital role on safety performance (Cox \& Cheyne, 2000). Involvement is a behavioural-approach that allows employees to be part of the decision-making process; this empowers workers by providing them with the responsibility of making vital decisions in setting goals (Vredenburgh, 2002).

A Finnish study Vartia (1996) discussed the scenario where managers or supervisors tended to solve conflicts in their own authoritarian way, causing a poor safety climate and promoting workplace bullying. Based on the reactance theory (e.g., Brehm \& Brehm, 1981), when workers felt supervisors or managers were unsupportive, they tend to not practice (or to resist) safety behaviours and rules in order to "get back" at the management (Kelloway \& Barling, 2010). Therefore, when employees believed that they had no voice when it came to the workplace's safety policies, they developed a sense of indifference.

Other studies revealed that when the management intended to make a decision which was related to personnel by listening (Singer \& Obach, 2013) and engaging personnel in the decision-making as 'a key role of involvement in the workplace', a positive association with employee motivation and psychological well-being was present (Felstead, Gallie, Green, \& Zhou, 2010). This research also discovered a positive correlation between role and involvement $(\mathrm{r}=0.151, \mathrm{p}<0.05)$. Involvement, collaboration and safety management were also factors of the safety climate (Hřivik, Tharaldsen, Baste, \& Moen, 2009).

The results displayed a correlation between work environment scores of the safety climate scale and relationship scores of the HSE scale. The relationship scores had a moderately positive, significant connection with the work environment factor $(r=0.329, \mathrm{p}<0.01)$. Safety climate played an essential role to improve psychosocial risks in the workplace. A Norwegian study demonstrated that bullying and harassment were associated with the social environment at work (Einarsen, 2000). When there was a poor work environment and a weak management style, there was a significant expectation of increased bullying (Agervold \& Mikkelsen, 2004; Einarsen, Raknes, \& Matthiesen, 1994; Salin \& Hoel, 2011). However, a satisfied attitude with the work environment contributed to high levels of a psychosocial safety climate. In the absence of psychological health and safety within a workplace, high rates of bullying and harassment were observed, which in turn, reduced the relationship scores (Salin, 2003).

When reviewing the mental health aspect of a safety climate in a workplace, a study on the occupational safety climate demonstrated that poor communication was a predictor of depressive symptoms (Cho et al., 2008). A small, yet significant, negative relationship between depression associated with personal priorities and the need for safety $(p<0.01)$ with the work environment $(p<0.05)$ was found. Improvements in the work environment may decrease the risk of depression symptoms (Wang, Schmitz, Dewa, \& Stansfeld, 2009). A study revealed that about $8 \%$ of depression may be explained by environmental factors in the workplace (Prüss-Üstün \& Corvalán). 
Williamson (2001) and Stansfeld and Candy (2006) found that if health and safety procedures were properly conducted, they may help identify work-related stress and recognise certain personnel groups that were more susceptible to stress. In addition, the psychosocial work environment models, such as Cox, Griffiths, and Rial-Gonzalwz (2000) and Dollard and Bakker (2010), illustrated that the psychosocial safety climate may affect mental health status.

A study by Hofmann and Stetzer (1996) regarding chemical processing workers discovered a positive relationship between workplace psychosocial risks and unsafe practices within the workplace. A study in China also presented an association between the organisational climate and job satisfaction in workers (Siu, Phillips, \& Leung, 2004). In accordance with the current research, a study on workers of oil and gas companies detected that the common stressor among employees and managers was poor communication. They concluded that good communication in the workplace may protect employees from the dangerous effects of further stressors, and this would contribute to improved safety (Brešić et al., 2007).

\subsection{Relationship between SCAT and HSE}

The results illustrated that psychosocial risk factors (HSE subscales) were positively related to workplace safety climate. Management commitment and work environment subscales of the safety climate were related to all HSE subscales. A large correlation was seen between work environment with demands $(\mathrm{r}=0.447)$, managers' support $(\mathrm{r}=0.359)$, relationships $(\mathrm{r}=0.329)$, role $(\mathrm{r}=0.212)$ and change $(\mathrm{r}=0.343, \mathrm{p}<0.01)$.

The results displayed a significant relationship between some psychosocial subscales and safety climate subscales in the company. It revealed that safety climate factors may influence psychosocial risks in the workplace and vice versa. Psychosocial risk factors, specifically demands, manager's support and relationships, influenced the attitude of employees in regards to the safety climate; especially for management commitment, communication and work environment.

The mitigation of workplace psychosocial risk factors is an important reason to develop health and safety policies to monitor the work environment; leading to the promotion of workplace mental health (Dollard et al., 2007; M. Dollard, Skinner, Tuckey, \& Bailey, 2007).

From a theoretical standpoint, Kahn and Byosiere (1992) proposed that work environment characteristics had an influence on producing role conflict in the workplace (Pomaki et al., 2007). Along these lines, a study found that psychological risks (such as high demand) may increase unsafe behaviour within the workplace (Siu et al., 2004).

A study in safety climate and supervisory behaviour demonstrated that if supervisors were not concerned about safety while they were interacting with subordinates, workers would not follow safely rules. They further found that supervisor's support of subordinates increased safety climate scores (Zohar \& Luria, 2003).

The results of the current research were in-line with another study that portrayed the lack of safety climate policies (or procedures that target improving job demands) tend to prevail for long periods within the workplace. A consequence of the lack of procedures to report work overload led to increased conflicts and relationship problems; especially when employees' concerns were not adhered to, or the importance of psychological well-being was not recognised (Dollard \& Bakker, 2010). Therefore, an enhanced safety climate aided employees to better cope with their job demands and relationships by the development of appropriate strategies.

PSC model indicated that the psychosocial safety climate and communication affected the psychosocial risks in the workplace. Recent studies confirmed that the psychosocial safety climate was able to clarify the roots of demand, supervisor's support, justice and other job resources (Dollard \& McTernan, 2011; Rickard et al., 2012). In addition, it was found that a positive psychosocial safety climate may control workplace bullying and harassment. It was clarified that a positive psychosocial safety climate may be a leading indicator for bullying, harassment, job resources and psychological health (Law, Dollard, Tuckey, \& Dormann, 2011).

Overall, this study supported other research findings. It confirmed that the positive attitude of employees to safety climate factors was closely linked to the HSE factors in the workplace. If the management had a commitment to health and safety procedures and safety rules, it can attain a potentially positively impact on psychosocial risks.

\section{Conclusion}

Overall, the need of policies about psychosocial risks and psychosocial safety climate to guide employers to promote health is concerning. Ethical policies for supporting proper workplace relationships may contribute to low level score of psychosocial risks at work. The study supported the possibility that employees' attitude to 
safety climate was correlated to psychosocial risks at the workplace. Employers should pay more attention to relationship role and communication factors. Safety climate factors may impact psychosocial risks in the workplace and vice versa.

\section{References}

Agervold, M., \& Mikkelsen, E. G. (2004). Relationships between bullying, psychosocial work environment and individual stress reactions. Work and Stress, 18(4), 336-351. http://dx.doi.org/10.1080/026783704123313 19794

Aust, B., \& Ducki, A. (2004). Comprehensive Health Promotion Interventions at the Workplace: Experiences With Health Circles in Germany. Journal of Occupational Health Psychology, 9(3), 258-270. http://dx.doi.org/10.1037/1076-8998.9.3.258

Baba, V. V., Galperin, B. L., \& Lituchy, T. R. (1999). Occupational mental health: a study of work-related depression among nurses in the Caribbean. International Journal of Nursing Studies, 36, 163-169. http://dx.doi.org/10.1016/S0020-7489(99)00002-4

Brešić, J., Knežević, B., Milošević, M., Tomljanović, T., Golubović, R., \& Mustajbegović, J. (2007). Stress and work ability in oil industry workers.

Carayon, P. (2007). Handbook of human factors and ergonomics in health care and patient safety. CRC.

Chang, E. M., Daly, J. W., Hancock, K. M., Bidewell, J., Johnson, A., Lambert, V. A., \& Lambert, C. E. (2006). The relationships among workplace stressors, coping methods, demographic characteristics, and health in Australian nurses. Journal of Professional Nursing, 22(1), 30-38. http://dx.doi.org/10.1016/j.profnurs.2005. 12.002

Chen, Wong, \& Yu. (2009). Mental health issues in Chinese offshore oil workers. Occupational Medicine-Oxford, 59(8), 545-549. http://dx.doi.org/10.1093/occmed/kqp118

Cheng, Y., Kawachi, I., Coakley, E. H., Schwartz, J., \& Colditz, G. (2000). Association between psychosocial work characteristics and health functioning in American women: prospective study. Bmj, 320(7247), 1432-1436. http://dx.doi.org/10.1136/bmj.320.7247.1432

Cho, J. J., Kim, J. Y., Chang, S. J., Fiedler, N., Koh, S. B., Crabtree, B. F., . . Choi, Y. H. (2008). Occupational stress and depression in Korean employees. International archives of occupational and environmental health, 82(1), 47-57. http://dx.doi.org/10.1007/s00420-008-0306-4

Choobineh, A., Movahed, M., Tabatabaie, S. H., \& Kumashiro, M. (2010). Perceived demands and musculoskeletal disorders in operating room nurses of Shiraz city hospitals. Industrial Health, 48(1), 74-84. http://dx.doi.org/10.2486/indhealth.48.74

Choudhry, R. M., Fang, D., \& Mohamed, S. (2007). The nature of safety culture: A survey of the state-of-the-art. Safety Science, 45(10), 993-1012. http://dx.doi.org/10.1016/j.ssci.2006.09.003

Cooper, C. L., \& Cartwright, S. (1994). Healthy mind; healthy organization-A proactive approach to occupational stress. Human Relations, 47(4), 455-471. http://dx.doi.org/10.1177/001872679404700405

Cooper, C. L., \& Sutherland, V. J. (1987). Job stress, mental health, and accidents among offshore workers in the oil and gas extraction industries. Journal of occupational medicine: official publication of the Industrial Medical Association, 29(2), 119.

Cox, \& Cheyne, A. (2000). Assessing safety culture in offshore environments. Safety Science, 34(1-3), 111-129.

Cox, Griffiths, A., \& Rial-Gonzalwz, E. (2000). Research on Work-related Stress. Retrieved from http://osha.europa.eu/en/publications/reports/203

Cox, Jones, B., \& Rycraft, H. (2004). Behavioural approaches to safety management within UK reactor plants. Safety Science, 42(9), 825-839. http://dx.doi.org/10.1016/j.ssci.2004.03.002

Cox, Leka, S., Ivanov, I., \& Kortum, E. (2004). Work, employment and mental health in Europe. Work and Stress, 18(2), 179-185. http://dx.doi.org/10.1080/02678370412331272651

Dollard, \& Bakker, A. B. (2010). Psychosocial safety climate as a precursor to conducive work environments, psychological health problems, and employee engagement. Journal of Occupational and Organizational Psychology, 83(3), 579-599.

Dollard, \& McTernan, W. (2011). Psychosocial safety climate: a multilevel theory of work stress in the health and community service sector. Epidemiology and Psychiatric Sciences, 20(04), 287-293. 
Dollard, Skinner, N., Tuckey, M., \& Bailey, T. (2007). National surveillance of psychosocial risk factors in the workplace: An International Overview Work and Stress, 21(1), 1-29. http://dx.doi.org/10.1080/026783707 01254082

Dollard, M., Skinner, N., Tuckey, M. R., \& Bailey, T. (2007). National surveillance of psychosocial risk factors in the workplace: An international overview. Work and Stress, 21(1), 1-29. http://dx.doi.org/10.1080/ 02678370701254082

Einarsen, S. (2000). Harassment and bullying at work: A review of the Scandinavian approach. Aggression and violent behavior, 5(4), 379-401. http://dx.doi.org/10.1016/S1359-1789(98)00043-3

Einarsen, S., Raknes, B. r. I., \& Matthiesen, S. B. (1994). Bullying and harassment at work and their relationships to work environment quality: An exploratory study. European Journal of Work and Organizational Psychology, 4(4), 381-401. http://dx.doi.org/10.1080/13594329408410497

Fairhurst, G. T. (1993). The leader - member exchange patterns of women leaders in industry: A discourse analysis. Communications Monographs, 60(4), 321-351. http://dx.doi.org/10.1080/03637759309376316

Fairhurst, G. T., \& Chandler, T. A. (1989). Social structure in leader-member interaction. Communications Monographs, 56(3), 215-239. http://dx.doi.org/10.1080/03637758909390261

Felstead, A., Gallie, D., Green, F., \& Zhou, Y. (2010). Employee involvement, the quality of training and the learning environment: an individual level analysis. The International Journal of Human Resource Management, 21(10), 1667-1688. http://dx.doi.org/10.1080/09585192.2010.500489

Fujiwara, K., Tsukishima, E., Tsutsumi, A., Kawakami, N., \& Kishi, R. (2003). Interpersonal conflict, social support, and burnout among home care workers in Japan. Journal of Occupational Health, 45(5), 313-320. http://dx.doi.org/10.1539/joh.45.313

Gardner, R. (2003). Overview and characteristics of some occupational exposures and health risks on offshore oil and gas installations. Annals of Occupational Hygiene, 47(3), 201-210. http://dx.doi.org/10.1093/ annhyg/meg028

Harnois, G., \& Gabriel, P. (2000). Mental health and work: impact, issues and good practices. WHO,ILO, 222.

Hinkka, K., Kuoppala, J., Väänänen-Tomppo, I., \& Lamminpää, A. (2013). Psychosocial work factors and sick leave, occupational accident, and disability pension: a cohort study of civil servants. Journal of Occupational and Environmental Medicine, 55(2), 191-197. http://dx.doi.org/10.1097/JOM.0b013e3182 $7943 \mathrm{fe}$

Hofmann, D. A., \& Stetzer, A. (1996). A cross - level investigation of factors influencing unsafe behaviors and accidents. Personnel Psychology, 49(2), 307-339. http://dx.doi.org/10.1111/j.1744-6570.1996.tb01802.x

Hřivik, D., Tharaldsen, J. E., Baste, V., \& Moen, B. E. (2009). What Is Most Important for Safety Climate: The Company Belonging or the Local Working Environment?A Study from the Norwegian Offshore Industry. Safety Science, 47(10), 1324-1331. http://dx.doi.org/10.1016/j.ssci.2009.04.001

HSE. (2004). In H. a. S. Executive (Ed.), HSE Management Standards Analysis Tool User Manual. London.

HSE. (2008). Working together to reduce stress at work - a guide for employees. In HSE (Ed.), Health and Safety Executive.

Inoue, \& Kawakami, N. (2010). Interpersonal Conflict and Depression among Japanese Workers with High or Low Socioeconomic Status: Findings from the Japan Work Stress and Health Cohort Study. Social Science \& Medicine, 71(1), 173-180.

Johnstone, R., Quinlan, M., \& McNamara, M. (2010). OHS inspectors and psychosocial risk factors: Evidence from Australia. Safety Science, 49(4), 547-557. http://dx.doi.org/10.1016/j.ssci.2010.09.016

Kazi, A., \& Haslam, C. O. (2013). Stress management standards: a warning indicator for employee health. Occupational Medicine-Oxford, 63(5), 335-340. http://dx.doi.org/10.1093/occmed/kqt052

Kelloway, E. K., \& Barling, J. (2010). Leadership development as an intervention in occupational health psychology. Work and Stress, 24(3), 260-279. http://dx.doi.org/10.1080/02678373.2010.518441

Kortum, E., Leka, S., \& Cox, T. (2010). Psychosocial risks and work-related stress in developing countries: health impact, priorities, barriers and solutions. International Journal of Occupational Medicine and Environmental Health, 23(3), 225-238. http://dx.doi.org/10.2478/v10001-010-0024-5

Landsbergis, P., Grzywacz, J. G., \& LaMontage, A. (2012). Work Organization, Job Insecurity, and Occupational 
Health Disparities: An Issue Paper for Discussion at the Eliminating Health and Safety Disparities at Work Conference, Chicago, Illinois, September 14 And 15, 2011.

LaRocco, J. M., House, J. S., \& French, J. R. P. Jr. (1980). Social support, occupational stress, and health. Journal of Health and Social Behavior, 202-218. http://dx.doi.org/10.2307/2136616

Law, R., Dollard, M. F., Tuckey, M. R., \& Dormann, C. (2011). Psychosocial safety climate as a lead indicator of workplace bullying and harassment, job resources, psychological health and employee engagement. Accident Analysis and Prevention. http://dx.doi.org/10.1016/j.aap.2011.04.010

Lee, Yeh, Chen, \& Wang. (2005). Prevalence and psychosocial risk factors of upper extremity musculoskeletal pain in industries of Taiwan: a nationwide study. Journal of Occupational Health, 47(4), 311-318. http://dx.doi.org/10.1539/joh.47.311

Leka, \& Jain. (2010). Health Impact of Psychosocial Hazards at Work: An Overview WHO.

LiWang, J., Schmitz, N., Dewa, C., \& Stansfeld, S. (2009). Changes in Perceived JobStrain and the Risk of Major Depression: Results From a Population-based Longitudinal Study. American Journal of Epidemiology, 169(9).

Loughborough University. (1998). Safety Climate Measurement User Guide and Toolkit.

Mearns, K., Whitaker, S. M., \& Flin, R. (2003). Safety climate, safety management practice and safety performance in offshore environments. Safety Science, 41(8), 641-680. http://dx.doi.org/10.1016/S09257535(02)00011-5

Mohamedi, F. (2011). The Oil and Gas Industry. Washington DC United States Institute of Peace. Retrieved from http://iranprimer.usip.org/resource/oil-and-gas-industry.

NIOC. (2012). NIOC at a Glance. Retrieved May, 2012, from http://www.nioc.ir/Portal/Home/Default.aspx? CategoryID=f398bd54-e170-44e9-a841-710c6c92b3a0\& TabNo=1

Nomura, K., Nakao, M., Sato, M., Ishikawa, H., \& Yano, E. (2007). The association of the reporting of somatic symptoms with job stress and active coping among Japanese white-collar workers. Journal of Occupational Health, 49(5), 370-375. http://dx.doi.org/10.1539/joh.49.370

Peiró Silla, J. M. (2000). Assessment of psychosocal risks and prevention strategies: the amigo model as the basis of the prevenlab/psicosocial methodology. Psychology in Spain, (4), 139-166.

Pomaki, G., Supeli, A., \& Verhoeven, C. (2007). Role conflict and health behaviors: Moderating effects on psychological distress and somatic complaints. Psychology and Health, 22(3), 317-335. http://dx.doi.org/10.1080/14768320600774561

Portuné, R. (2012). Psychosocial Risks in the Workplace: An Increasing Challenge for German and International Health Protection.

Press.TV. (2012). Iran's annual gas revenues from South Pars to reach $\$ 100 \mathrm{bn}$ : Official.

Productivity Commission. (2010). Performance Benchmarking of Australian Business Regulation: Occupational Health \& Safety. Research Report, Canberra: The Productivity Commission Australian Government.

Prüss-Üstün, A., \& Corvalán, C. (2006). Preventing disease through healthy environments. World Health Organization Geneva.

Rickard, G., Lenthall, S., Dollard, M., Oppie, T., Knight, S., Dunn, S., ... Brewester-Webb, D. (2012). Organisational intervention to reduce occupational stress and turnover in hospital nurses in the Northern Territory, Australia. Collegian: Journal of the Royal College of Nursing Australia.

Saijo, Y., Ueno, T., \& Hashimoto, Y. (2008). Twenty - four - hour shift work, depressive symptoms, and job dissatisfaction among Japanese firefighters. American journal of industrial medicine, 51(5), 380-391. http://dx.doi.org/10.1002/ajim.20571

Salin, D. (2003). Ways of explaining workplace bullying: A review of enabling, motivating and precipitating structures and processes in the work environment. Human Relations, 56(10), 1213-1232. http://dx.doi.org/10.1177/00187267035610003

Salin, D., \& Hoel, H. (2011). Organisational causes of workplace bullying. In Bullying and harassment in the workplace: Developments in theory, research, and practice (pp. 227-243).

Samra, J., Gilbert, M., Shain, M., \& Bilsker, D. (2009). Psychosocial Risk Factors. Guarding Mind at Work. 
Schmidt, S., Roesler, U., Kusserow, T., \& Rau, R. (2014). Uncertainty in the workplace: Examining role ambiguity and role conflict, and their link to depression-a meta-analysis. European Journal of Work and Organizational Psychology, 23(1), 91-106. http://dx.doi.org/10.1080/1359432X.2012.711523

Sias, P. M. (2005). Workplace relationship quality and employee information experiences. Communication Studies, 56(4), 375-395. http://dx.doi.org/10.1080/10510970500319450

Singer, M., \& Obach, J. J. (2013). Listening to workers: The overtime versus hiring dilemma. Journal of Business Research. http://dx.doi.org/10.1016/j.jbusres.2013.01.009

Siu, O.-L., Phillips, D. R., \& Leung, T.-W. (2004). Safety climate and safety performance among construction workers in Hong Kong: The role of psychological strains as mediators. Accident Analysis and Prevention, 36(3), 359-366. http://dx.doi.org/10.1016/S0001-4575(03)00016-2

Stansfeld, S., \& Candy, B. (2006). Psychosocial work environment and mental health-a meta-analytic review. Scandinavian Journal of Work Environment Health, 32 (6 Special issue), 443-462.

Tsuno, K., Kawakami, N., Inoue, A., Ishizaki, M., Tabata, M., Tsuchiya, M., ... Shimazu, A. (2009). Intragroup and intergroup conflict at work, psychological distress, and work engagement in a sample of employees in Japan. Industrial Health, 47(6), 640-648. http://dx.doi.org/10.2486/indhealth.47.640

Vartia, M. (1996). The sources of bullying-psychological work environment and organizational climate. European Journal of Work and Organizational Psychology, 5(2), 203-214. http://dx.doi.org/10.1080/135943 29608414855

Vredenburgh, A. G. (2002). Organizational safety: Which management practices are most effective in reducing employee injury rates? Journal of Safety Research, 33(2), 259-276. http://dx.doi.org/10.1016/S00224375(02)00016-6

Wang, \& Schmitz. (2011). Does job strain interact with psychosocial factors outside of the workplace in relation to the risk of major depression? The Canadian National Population Health Survey. Social psychiatry and psychiatric epidemiology, 46(7), 577-584.

Williamson, A. M. (2001). Managing stress in the workplace" Part II - The scientific basis (knowledge base) for the guide. In N. I. f. O. H. a. Safety (Ed.).

Ylikoski. (2008). Promoting and Maintaining Workability (PMWA) as an Approach on Well-being at Work for an Aging Workforce in Finland Finnish Institute of Occupational Health. Finland: Finnish Institute of Occupational Health.

Yu, S., Gu, G., Zhou, W., \& Wang, S. (2008). Psychosocial work environment and well-being: a cross-sectional study at a thermal power plant in China. Journal of Occupational Health, 50(2), 155-162. http://dx.doi.org/10.1539/joh.L7098

Zohar. (2010). Thirty years of safety climate research: Reflections and future directions. Accident Analysis and Prevention, 42(5), 1517-1522.

Zohar, \& Luria, G. (2003). The use of supervisory practices as leverage to improve safety behavior: A cross-level intervention model. Journal of Safety Research, 34(5), 567-577.

\section{Copyrights}

Copyright for this article is retained by the author(s), with first publication rights granted to the journal.

This is an open-access article distributed under the terms and conditions of the Creative Commons Attribution license (http://creativecommons.org/licenses/by/3.0/). 